\title{
CARACTERIZAÇÃO DOS UTILIZADORES DE PISCINAS EM PORTUGAL: PRÁTICAS USUAIS E MOTIVAÇÕES
}

\author{
Henrique Pereira Neiva ${ }^{1,2}$, Luís Brandão Faíl ${ }^{1}$, António José Silva, ${ }^{2,3}$ Daniel \\ Almeida Marinho ${ }^{1,2}$ \\ ${ }^{1}$ Universidade da Beira Interior, Covilhã (Portugal) \\ ${ }^{2}$ Centro de Investigação em Desporto, Saúde e Desenvolvimento Humano, CIDESD (Portugal) \\ ${ }^{3}$ Universidade de Trás-os-Montes e Alto Douro, Vila Real (Portugal)
}

OPEN ACCES

Correspondencia Daniel Almeida Marinho Convento de Santo António, 6201 001 Covilhã dmarinho@ubi.pt 00351275329153

Funciones de los autores: Todos los autores trabajaro equitativamente en consecución del trabajo

Recibido: $1 / 5 / 2018$ Aceptado: $21 / 8 / 2018$ Publicado: $31 / 1 / 2019$

Citación:

Neiva, H., Faíl, L, Silva, A. J., \& Marinho, D. (2019). Caracterização dos utilizadores de piscinas em Portugal: práticas usuais e motivações. RIAA. Revista de Investigación en Actividades Acuáticas, $3(5), 3-7$

https://doi.org/10.21134/riaa.v3i5.1635

@creative

(c) (1)(2) (2)

Creative Commons License Esta obra está bajo una licencia de Creative Commons ReconocimientoNoComercial-Compartir-Igua 4.0 Internacional
Contexto: A realização de exercício em contexto aquático apresenta numerosos benefícios. Contudo, a sua prática pode ser condicionada por alguns constrangimentos.

Objetivos: Pretendemos assim verificar quais os hábitos e as motivações dos portugueses relativamente à utilização das piscinas, procurando assim perceber as condicionantes para a prática do exercício físico em contexto aquático.

Método: Foi aplicado um inquérito a 530 indivíduos, utilizadores de piscinas cobertas, de ambos os sexos, com idades compreendidas entre os 18 e os 88 anos de idade. O questionário incluía informações sobre a caraterização pessoal e também acerca da realização de atividades aquáticas por parte de cada indivíduo. Os dados resultantes foram posteriormente analisados por métodos estatísticos descritivos.

Resultados: Verificou-se uma prevalência dos indivíduos para a prática de natação (62\%) seguido da hidroginástica (35\%), sendo motivados na sua grande parte pela procura da melhoria da saúde (47\%). Na sua maioria, os sujeitos são experientes, frequentando a piscina há mais de 2 anos

Conclusoes: Observou-se que os portugueses avaliados praticam essencialmente natação e hidroginástica, ao final do dia e motivados pela melhoria da saúde e do bem-estar individual.

Palavras-chave: Exercício, atividade aquática, aptidão física, inquérito, motivação.

Título: Caracterización de los usuarios de piscinas en Portugal: las prácticas y motivaciones habituales.

Antecedentes: La realización de ejercicio en contexto acuático presenta numerosos beneficios. Sin embargo, su práctica puede estar condicionada por algunas limitaciones.

Objetivos: Tenemos la intención de comprobar cuáles son los hábitos y motivaciones de los portugueses sobre el uso de las piscinas, buscando de esta forma de entender las condiciones para la práctica de ejercicio físico en el medio acuático. Método: Se aplicó una encuesta a 530 individuos, usuarios de piscinas cubiertas, de ambos sexos, de edades comprendidas entre los 18 y los 88 años. El cuestionario incluía información sobre la caracterización personal y también acerca de la realización de actividades acuáticas por parte de cada individuo. Los datos resultantes fueron posteriormente analizados por métodos estadísticos descriptivos.

Resultados: Se verificó una prevalencia de los individuos para la práctica de natación (62\%) seguida de los aeróbicos en el agua (35\%), siendo motivados en gran parte por la demanda de la mejora de la salud (47\%). En su mayoría, los sujetos son experimentados, frecuentando la piscina desde hace más de 2 años (64\%), 2 veces por semana $(60 \%)$, y con horario preferencial de las $18 \mathrm{~h}$ a las $22 \mathrm{~h}(50 \%)$

Conclusiones: Se observó que el evaluado portugués esencialmente practicar natación y aeróbicos en el agua al final del día, motivados por la mejora de la salud y el bienestar individual.

Palabras clave: Ejercicio, actividad acuática, aptitud física, encuesta, motivación

Title: Characcterization of pool users in Portugal: User practices and motivations.

Background: Exercising in an aquatic context has numerous benefits. However, the in-water practices may be inhibited by some constraints.

Objectives: We intend to verify the habits and motivations of the Portuguese people regarding the use of swimming pools, thus trying to understand the influencing factors for the practice of aquatic physical exercise.

Method: 530 participants, indoor swimming pool users, of both genders, aged 18-88 years, answered to a survey individually. The survey included information on personal characterization and also on the practices that were usual in aquatic context by each individual. The resulting data were then analyzed using descriptive statistical methods.

Results: There was a prevalence for swimming $(62 \%)$ followed by water aerobics $(35 \%)$ regarding the usual aquatic practices, being motivated mostly by health improvement $(47 \%)$. Most of the subjects were experienced, attending the swimming pool for more than 2 years $(64 \%)$, twice a week $(60 \%)$, and preferential hours from 6:00 p.m. to 10:00 p.m. (50\%).

Conclusions: It was observed that the Portuguese swimming pool users choose preferentially to swim and to perform water aerobics, at the end of the day and motivated by the improvement of health and individual well-being. Key words: Exercise, aquatic activity, fitness, survey, motivations. 


\section{Introdução}

O sedentarismo é uma das grandes problemáticas que se enfrenta nos dias de hoje, tornando cada vez mais importante o seu combate (Neiva, Faíl, Izquierdo, Marques \& Marinho, 2018). Neste sentido, a prática de exercício físico, pelos seus benefícios, tem sido cada vez mais apontada como um dos principais métodos de oposição ao sedentarismo em qualquer faixa etária (Neiva et al., 2018). Sabe-se que o exercício físico é fundamental para atenuar os problemas ao nível da saúde e da capacidade funcional, no entanto, parecem existir situações em que a sua prática pode estar condicionada. De facto, as limitações que surgem em indivíduos com níveis baixos de aptidão física, com dificuldades de locomoção, como em casos de obesidade e/ou em idosos, e por modificações fisiológicas e/ou estruturais que se vão gerando, podem impossibilitar ou limitar a prática de determinadas atividades (Neiva et al., 2018; Tsourlou, Benik, Dipla, Zafeiridis \& Kellis, 2006).

De modo a facilitar a prática de exercício físico por todos, tem sido observado um aumento significativo da sua realização em contexto aquático, tanto em indivíduos que apresentem alguma debilidade, a nível físico ou mental, como em atletas em processo de recuperação de lesões, podendo assim servir inclusivamente como um método alternativo para manter a prática de exercício físico(Borreani et al., 2015; Colado, Tella, Triplett \& González, 2009; Neiva et al., 2018). O meio aquático tem vindo a ser utilizado como uma alternativa segura e válida de exercício em relação ao exercício terrestre essencialmente devido às propriedades que o meio oferece, a referir especificamente a flutuabilidade, que proporciona uma redução do efeito do peso corporal sobre as articulações e reduz as forças de compressão nas articulações, e as forças de arrasto aumentadas, que possibilitam a carga externa durante a maioria dos movimentos (Borreani et al., 2015; Takeshima et al., 2002; Tsourlou et al., 2006). De um modo geral, estas propriedades podem ser benéficas quer para pessoas saudáveis e sem qualquer limitação, quer para indivíduos com diferentes tipos de limitações físicas ou fragilidades (Borreani et al., 2015; Colado et al., 2009).

Apesar da maioria das pessoas conhecer os efeitos benéficos que advêm da prática de exercício físico, por vezes a sua prática pode ser condicionada por algumas barreiras, as quais, se não forem percepcionadas, podem representar um obstáculo à adesão e manutenção em programas de exercício físico (Booth, Bauman, Owen \& Gore, 1997). Isto tem levado a um aumento da investigação com vista a identificar os principais condicionalismos que limitam as pessoas de praticar exercício físico (Myers \& Roth, 1997; Neiva et al., 2018). Torna-se assim fundamental conhecer os principais fatores que motivam as pessoas a tornarem-se fisicamente ativos, sobretudo no que diz respeito à realização de exercícios em meio aquático, assim como a manter esses níveis de atividade, uma vez que este conhecimento é importante para auxiliar a planear intervenções efetivas de atividade física (Dishman, Sallis \& Orenstein, 1985; Neiva et al., 2018). Desta forma, o presente estudo teve como principal objetivo verificar e descrever os hábitos de utilização das piscinas em Portugal, tentando perceber o que normalmente condiciona e motiva os indivíduos à realização de exercício físico em contexto aquático.

\section{Método}

\section{Participantes}

A amostra foi constituída por 530 indivíduos com idades compreendidas entre os 18 e os 88 anos de idade $(41.00 \pm 20.81$ anos de idade), de ambos os sexos ( $56 \%$ do sexo feminino e $44 \%$ do sexo masculino). Como critério de inclusão os indivíduos teriam que ser utilizadores de uma piscina coberta em Portugal e apresentar idades iguais ou superiores a 18 anos. Foram incluídos no estudo as pessoas que se enquadraram no perfil da amostra, que estiveram presentes nos dias determinados para a realização dos inquéritos e que concordaram voluntariamente em participar no estudo, mediante assinatura do termo de consentimento informado. Todos os procedimentos seguiram as recomendações da Declaração de Helsínquia e aprovados pela comissão de ética. As caraterísticas dos sujeitos encontram-se na Tabela 1.

Tabela 1. Valores médios ( \pm desvio-padrão) da idade, altura e massa corporal dos sujeitos pertencentes à amostra.

\begin{tabular}{lc}
\hline & Sujeitos \\
\hline Idade (anos) & $41.00 \pm 20.81$ \\
Altura (m) & $1.68 \pm 0.10$ \\
Massa Corporal (kg) & $71.05 \pm 12.03$ \\
\hline
\end{tabular}

\section{Medidas}

Como intrumento de avaliação foi utilizado um inquérito composto por 20 questões. Estas questões eram divididas em duas partes, sendo que na primeira parte foram coletados dados sobre a caracterização pessoal, nomeadamente do ano de nascimento, género, peso, altura, profissão, área de residência e habilitações académicas. Na segunda parte foram colocadas perguntas de resposta fechada sobre o tipo de atividades aquáticas realizado, nomeadamente, experiência e há quanto tempo frequenta a piscina, frequência semanal e anual de utilização, horário preferêncial, tipo de atividades praticadas e acompanhamento profissional, motivos para a prática e, intensidade dedida ao exercício físico aquático.

\section{Procedimento}

Um estudo transversal foi realizado em piscinas cobertas de várias cidades portuguesas. Um inquérito anónimo foi aplicado em cada local por um período de vários dias (5 dias), de forma a incluir o máximo possível de indivíduos utilizadores das piscinas, desde que cumprissem os critérios de inclusão. Os inquéritos foram apresentados a todos os indivíduos que entravam nas piscinas e que aceitavam participar no estudo, juntamente com outra folha que descrevia o estudo, contendo também a declaração de confidencialidade dos dados pessoais.

Foram distribuídos um total de 530 inquéritos validados através dos critérios de inclusão. Tal como previamente referido, o inquérito era composto por 20 questões, sendo dividido em duas partes, uma primeira parte dedidacada à caraterização pessoal e uma segunda parte do inquérito englobou questões relacionadas com a prática de atividades aquáticas, particularmente sobre o tipo de atividades aquáticas realizado, o tempo que pratica este tipo de atividades, a frequência semanal, o horário preferencial e as motivações para a sua realização.

\section{Análise estatística}

Para a análise dos dados foi utilizado o programa Microsoft Office Excel 16 e o programa de análise estatística Statistical Package of Social Science (SPSS) 22.0, ambos para Windows. O cálculo de médias, desvios-padrão foram realizados por métodos estatísticos padronizados, assim como foram realizadas representações gráficas para melhor interpretação dos resultados obtidos.

\section{Resultados}

Na Figura 1 podemos observar os valores obtidos relativamente às habilitações académicas por parte dos sujeitos. Dentre os frequentadores de instalações aquáticas, verificamos uma percentagem superior de pessoas que concluíram o secundário, seguindo-se os indivíduos que completaram um curso superior, os que 
têm apenas educação básica e quem nunca estudou. Para além destas informações, também se verificou que $56 \%$ da amostra eram estudantes, $30 \%$ eram pessoas reformadas, correspondendo os restantes $14 \%$ a outras profissões.

Na Figura 2 verifica-se o tempo com que os sujeitos utilizam as piscinas em Portugal, notando-se que a maioria pratica atividades em contexto aquático há mais de 2 anos, enquanto os restantes praticam há menos de 1 ano e entre 1 e 2 anos.

Figura 1. Habilitações académicas dos participantes.

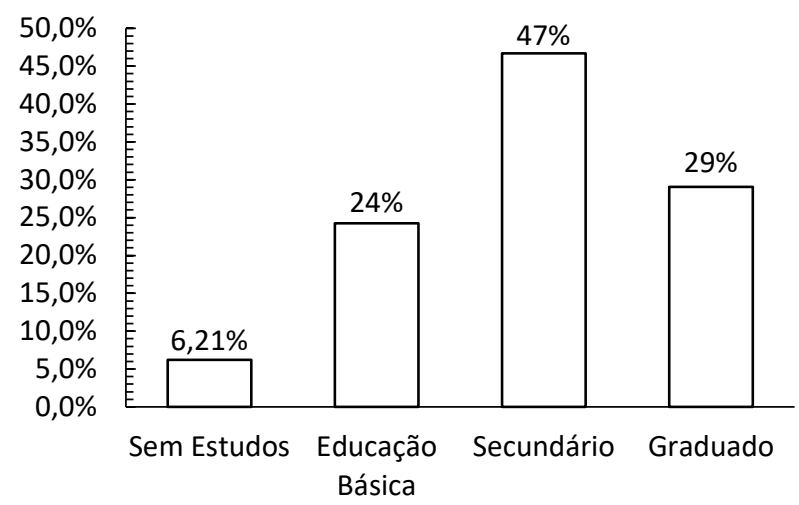

Figura 2. Experiência de utilização das piscinas.

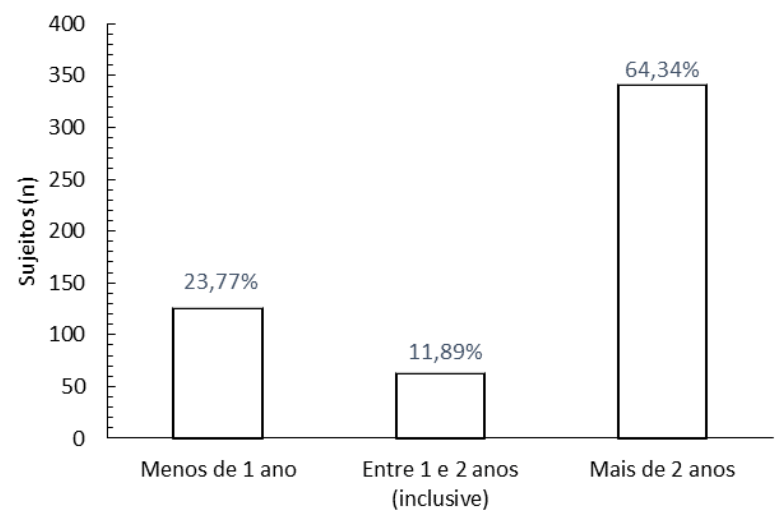

Pode observar-se na representação gráfica da Figura 3 a frequência com que os sujeitos praticam as atividades aquáticas na piscina semanalmente. A grande generalidade das pessoas participantes no estudo pratica entre 1 a 2 vezes por semana. Em menor quantidade são aquelas que indicaram praticar 3 vezes por semana, sendo mesmo poucos aqueles que referiram frequentar as piscinas mais do que 3 vezes por semana.

Figura 3. Frequência de utilização semanal das piscinas.

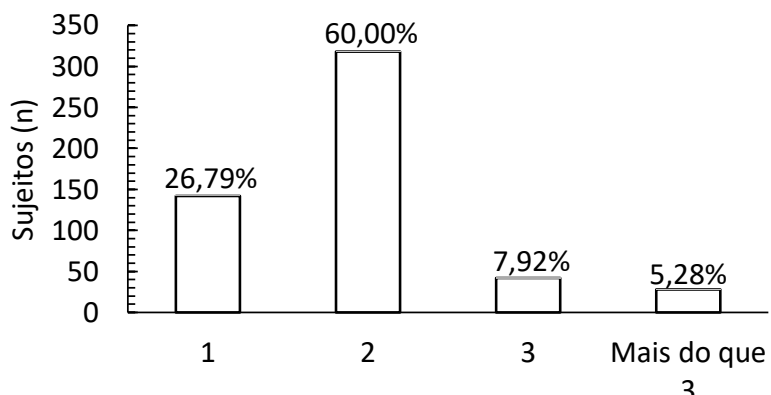

A Figura 4 demonstra qual o horário com que os indivíduos preferencialmente utilizam as piscinas. A maior parte das pessoas referem utilizar as piscinas durante o horário das $18 \mathrm{~h}-22 \mathrm{~h}$, sendo que cerca de um terço dos participantes frequentam entre as $8 \mathrm{~h}$ e as $12 \mathrm{~h}$, e menos ainda os que frequentam no período de horário $12 \mathrm{~h}-18 \mathrm{~h}$

Figura 4. Horário preferencial de utilização das piscinas.

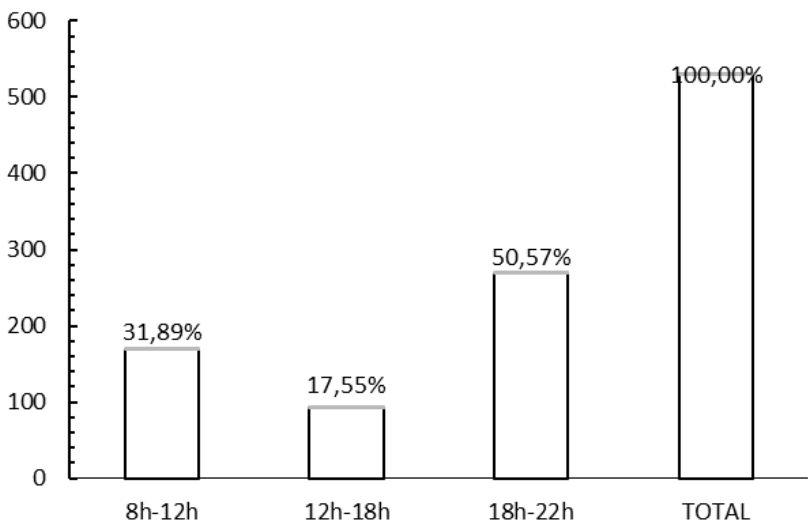

É possível observar-se na Figura 5 qual o tipo de prática de exercício que as pessoas fazem das piscinas. A maior parte dos frequentadores optam por praticar natação, seguido pelas aulas de hidroginástica. As aulas de hidroterapia são as menos frequentadas.

Figura 5. Atividades aquáticas praticadas nas piscinas.

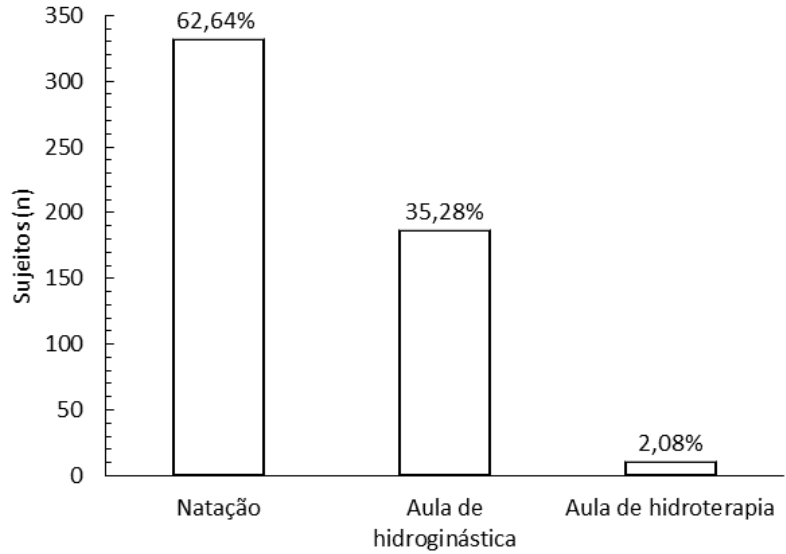

Por último, através da Figura 6 podemos observar as principais motivações que levam as pessoas a realizar exercício físico, particularmente atividades aquáticas. É de notar a importância que as pessoas dão à melhoria da saúde e à melhoria da condição física, não dando tanta relevância ao alívio do stress, à melhoria da coordenação motora e ao convívio social.

\section{Discussão}

O principal propósito do presente estudo foi descrever e conhecer os hábitos dos portugueses no que diz respeito à utilização de piscinas, procurando perceber também as preferências e as condicionantes que normalmente levam as pessoas a não pertencerem a programas de atividade física. Procurou-se também perceber os motivos que induzem os indivíduos à prática do exercício físico em contexto aquático.

Através dos resultados obtidos, pudemos perceber que a população portuguesa que habitualmente frequenta as piscinas para praticar 
algum tipo de atividade física aquática, pode ser caraterizada como pessoas instruídas, tendo a maioria concluído o secundário e sendo ainda estudantes ou então estando reformados. De notar ainda que estes utentes são essencialmente praticantes de natação e hidroginástica, frequentando os programas acompanhados por professores há mais de 2 anos. Podemos desta forma referir que a maior parte dos utilizadores das instalações aquáticas são experientes e regulares, denotando-se assim alguma satisfação e concretização pessoal na frequência regular da piscina. Nos nossos resultados verificamos ainda que parece prevalecer a prática regular de 2 vezes por semana, com horário ao final do dia (entre as $18 \mathrm{~h}$ e as $22 \mathrm{~h}$ ). A motivação para esta prática usual tem como razões fundamentais a melhoria da saúde e a melhoria da condição física, sendo pouco referido o alívio do stress, a melhoria da coordenação motora e pelo convívio social.

\section{Figura 6. Motivações para a prática de atividades aquáticas.}

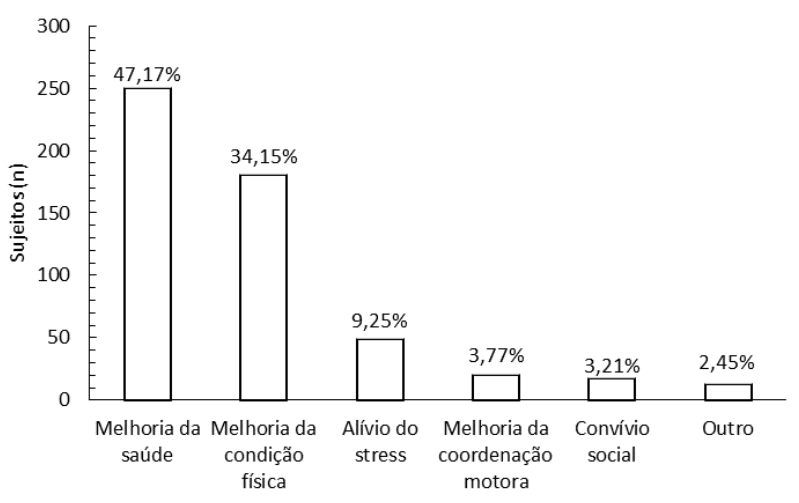

Algumas investigações corroboram com os resultados obtidos, realçando que a natação e a hidroginástica têm-se destacado cada vez mais como as principais modalidades praticadas em meio aquático, considerando os idosos os principais praticantes de hidroginástica (Lopes, Streit, Medeiros \& Mazo, 2014). Estes mesmos autores salientaram que a prática regular de hidroginástica e natação é altamente recomendada a idosos devido ao contributo que tem nesta faixa etária, uma vez que facilita a locomoção, diminuindo o impacto nas articulações e nos ossos, que devido ao processo de envelhecimento estão mais fragilizados (Lopes, Streit, Medeiros \& Mazo, 2014).

Também se observou uma prevalência por parte de estudantes e de pessoas reformadas para a prática destas atividades realizadas em piscinas, o que mostra uma maior utilização das piscinas por indivíduos mais jovens e por idosos. Estes resultados vão de encontro a alguns estudos que afirmaram que a prática de exercício físico devese iniciar quando somos novos, de forma a facilitar a adesão a programas de exercício na vida adulta e posteriormente (Telama, Yang, Laakso \& Viikari, 1997). Por outro lado, ao contrário dos nossos achados, onde se verificou um aumento da realização de exercício em jovens e idosos, havendo um equilíbrio da sua prática nos dois géneros, o estudo de Stephens e Caspersen (1994) mostrou que com o passar da idade, a realização de atividade física diminui, sobretudo nas mulheres idosas (Casperson, Merritt \& Stephens, 1994). Apesar da realização de exercício físico ser fundamental, o caso dos idosos é descrito como sendo o grupo etário mais difícil de conseguir incentivar a tornarem-se fisicamente ativos, uma vez que geralmente estes acreditam ser demasiado frágeis para praticar exercício, não o vendo como um dos recursos para o retardar do envelhecimento, acabado por abandonar a sua prática (Dishman, 1994).

A realização de exercício físico por vezes pode ser condicionada por determinados fatores, pelo que se torna fundamental, identificar as variáveis que podem afetar a adesão ao exercício (Schutzer \& Graves,
2004). Estas variáveis foram investigadas em vários estudos anteriores, tendo chegado à conclusão que as barreiras à realização de exercício podem variar consoante o género e as diferentes faixas etárias (Booth et al., 1997). Em adultos, foram consideradas como sendo as principais barreiras a falta de tempo, a falta de um parceiro para praticar exercício, problemas de saúde, custo financeiro e a falta de acesso a instalações adequadas (Godin et al., 1994). Por outro lado, em idosos, os motivos para a não realização de exercício foram a incapacidade física e a falta de vontade (Booth et al., 1997), sendo considerados o grupo etário mais predisposto a serem inativos e sem intenção de se tornarem ativos (Donovan \& Owen, 1994). Para além disto, também se observou que em mulheres o fator mais descrito é a incapacidade física (Booth et al., 1997).

Apesar destas variáveis referidas servirem como barreiras para a prática de atividades, algumas podem também estar relacionadas com as variáveis motivacionais, como é o caso dos problemas de saúde. Estes tanto podem restringir a capacidade de realizar exercício, como podem desempenhar um papel relevante ao servir de motivador para pessoa aumentar a realização de atividade física (Cohen-Mansfield, Marx \& Guralnik, 2003). Neste sentido, segundo a literatura, as principais motivações que levam à prática de exercício físico são o combate aos problemas de saúde, e consequentemente os benefícios para os parâmetros relacionados com a saúde, o gosto pela prática, o convívio social e a indicação médica (Lopes et al., 2014), entrando em concordância com alguns nossos resultados. Sabe-se que, as atividades em meio aquático, e de forma específica aquelas preferencialmente escolhidas pelos participantes no presente estudo, natação e hidroginástica, contribuem para melhoria da saúde e do bem-estar (Benassi \& Shigunov, 1993; Neiva et al., 2018).

Apesar da saúde ser um dos motivos elementares da maioria das pessoas para iniciar a realização de exercício, confirmado pelos dados do presente estudo, tal não acontece com as faixas etárias inferiores às nossas, não sendo considerado como aspeto fundamental para a adesão num programa de atividade física em adolescentes (Alves, Junger, Palma, Monteiro \& Resende, 2007). Devemos estar cientes que provavelmente não será a razão para integrar o exercício físico em idades mais novas, mas poderá ser motivo para a permanência na prática à medida que a idade vai avançando. Este facto é comprovado pelo motivo mais apontado no nosso estudo bem como pela experiência superior a dois anos relatada pelos participantes, o que faz denotar uma elevada taxa de participação e permanência na prática de atividades aquáticas. Normalmente o maior obstáculo à prática ocorre após 6 meses do início da prática, com a desistência de aproximadamente $50 \%$ dos indivíduos antes de identificarem qualquer melhoria na saúde (Dishman, 1994). O estudo de Massetto (2011) acrescenta que somente após cerca de 4 anos do começo da realização de algum tipo de atividade, o indivíduo adquire o hábito de praticar exercício através da sua incorporação no quotidiano. Seria assim interessante procurar acompanhar os participantes no nosso estudo e perceber a manutenção e os seus hábitos nos próximos anos. Sabemos que, neste aspeto, o papel do professor, a empatia criada e o reconhecimento da sua qualidade profissional são fatores a ter em conta para a motivação e prática regular de exercício físico aquático (Alves et al., 2007).

Investigações futuras devem utilizar os resultados obtidos e procurar perceber de melhor forma o acompanhamento realizado na prática de exercício em meio aquático, nomeadamente na natação e hidroginástica, visto serem as modalidades mais praticadas atualmente neste contexto. Deve haver cada vez mais a preocupação de realizar estudos de caráter longitudinal, com diferentes faixas etárias, e que relacionem o tempo de prática aos motivos de adesão e de permanência em programas de exercício, de modo a promover a prática de atividade física regular. Para além disto, também é necessário aumentar o número de investigações percebendo os 
fatores limitadores ao exercício em diferentes contextos, de forma a conseguir proporcionar estratégias para contornar essas condicionantes.

\section{Conclusões}

Os resultados obtidos permitiram perceber que a utilização das piscinas em Portugal é feita preferencialmente com a finalidade de praticar natação e hidroginástica, sendo que os praticantes são essencialmente motivados por parâmetros associados com a saúde e a melhoria da condição física. A maior parte dos utilizadores são regulares, experientes e procuram as instalações ao final do dia, provavelmente devido às limitações das ocupações diárias ou da oferta das instalações.

\section{Contribuição e implicações práticas}

As evidências do presente estudo permitem perceber os hábitos apresentados pela grande generalidade dos portugueses para a realização de atividades aquáticas em piscinas, assim como as suas motivações para a prática, facilitando assim a criação de estratégias que incentivem à prática de atividades aquáticas. Os profissionais interessados na prática de atividades aquáticas devem perceber que a população prefere recorrer à natação e hidroginástica, realizadas ao final do dia, com o intuito de melhorar a saúde, bem-estar e condição física. Dessa forma, importa tornar a oferta mais abrangente e talvez procurar estimular a procura de horários diferentes, modalidades/vertentes diferentes, e incentivando a motivações externas diferentes para faixas etárias mais novas.

\section{Agradecimentos}

Este projeto contou com o apoio da Fundação para a Ciência e Tecnologia e da União Europeia (UID/DTP/04045/2013; POCI-01-0145FEDER-006969) e também através do projeto NanoSTIMA - Macro-toNano Human Sensing: Rumo ao monitoramento e análise integrados de saúde multimodal (NORTE-01-0145-FEDER-000016).

\section{References}

Alves, M. P., Junger, W. L., Palma, A., Monteiro, W. D., \& Resende, H. G. D. (2007). Motivos que justificam a adesão de adolescentes à prática da natação: qual o espaço ocupado pela saúde. Revista Brasileira de Medicina do Esporte, 13(6), 421-426.

Benassi, S., \& Shigunov, V. (1993). Os motivos sociais e a sua relação com a prática da natação. Revista Brasileira de Ciências do Esporte, 15, 134.

Booth, M. L., Bauman, A., Owen, N., \& Gore, C. J. (1997). Physical activity preferences, preferred sources of assistance, and perceived barriers to increased activity among physically inactive australians. Preventive Medicine, 26(1), 131-137. https://doi.org/10.1006/pmed.1996.9982.

Borreani, S., Colado, J. C., Calatayud, J., Pablos, C., Moya-Nájera, D., \& Triplett, N. T. (2014). Aquatic resistance training: Acute and chronic effects. Strength \& Conditioning Journal, 36(3), 48-61.

Casperson, C. J., Merritt, R. K., \& Stephens, T. (1994). International physical activity patterns: a methodological perspective. In R.K. Dishman (Ed.), Advance in exercise adherence (pp. 73-110). Champaign, IL, England: Human Kinetics Publishers.

Cohen-Mansfield, J., Marx, M. S., \& Guralnik, J. M. (2003). Motivators and barriers to exercise in an older community-dwelling population. Journal of Aging and Physicl Activity, 11, 242- 53. https://doi.org/10.1123/japa.11.2.242.

Colado, J. C., Tella, V., Triplett, N. T., \& González, L. M. (2009). Effects of a short-term aquatic resistance program on strength and body composition in fit young men. The Journal of Strength \&
Conditioning Research, 23(2)

https://doi.org/10.1519/jsc.0b013e31818eff5d.

549-559.

Dishman, R. K (1994). Motivating older adults to exercise. Southern Medical Journal, 87, S79- 82.

Dishman, R. K., Sallis, J. F., \& Orenstein, D. R. (1985). The determinants of physical activity and exercise. Public Health Reports, 100, 158171.

Donovan, R. J., \& Owen, N. (1994). Social marketing and population interventions. In R.K. Dishman (Ed.), Advance in exercise adherence (pp. 249-90). Champaign, IL, England: Human Kinetics Publishers.

Godin, G., Desharnais, R., Valois, P., Lepage, L., Jobin, J., \& Bradet, R. (1994). Differences in perceived barriers to exercise between high and low intenders: observations among different populations. American Journal of Health Promotion, 8, 279-85.

Lopes, A. L., Streit, I. A., Medeiros, P. A., \& Mazo, G. Z. (2014). Motivos de ingresso e permanência de idosos em um programa de atividades aquáticas: um estudo longitudinal. Journal of Physical Education, 25(1),

23-32. https://doi.org/10.4025/reveducfis.v25i1.20371.

Massetto, S. T. (2011). Manutenção da prática de atividades aquáticas: tempo de prática e alterações dos principais motivos, na percepção dos idosos. Revista Mackenzie de Educação Física e Esporte, 10(1), 76-88.

Myers, R. E., \& Roth, D. L. (1997). Perceived benefits of and barriers to exercise and stage of exercise adoption in young adults. Health Psychology, 16, 277-83.

Neiva, H. P., Faíl, L. B., Izquierdo, M., Marques, M. C., \& Marinho, D. A. (2018). The effect of 12 weeks of water-aerobics on health status and physical fitness: An ecological approach. PloS one, 13(5). https://doi.org/10.1371/journal.pone.0198319.

Schutzer, K. A., \& Graves, B. S. (2004). Barriers and motivations to exercise in older adults. Preventive medicine, 39(5), 1056-1061.

Stephens, T., \& Caspersen, C. J. (1994). The demography of physical activity. In C. Bouchard, R. J. Shephard, \& T. Stephens (Eds.), Physical activity, fitness, and health: International proceedings and consensus statement (pp. 204-13). Champaign, IL, England: Human Kinetics Publishers.

Takeshima, N., Rogers, M. E., Watanabe, E., Brechue, W. F., Okada, A., Yamada, T., ... \& Hayano, J. (2002). Water-based exercise improves health-related aspects of fitness in older women. Medicine \& Science in Sports \& Exercise, 34(3), 544-551. https://doi.org/10.1097/00005768-200203000-00024.

Telama, R., Yang, X., Laakso, L., \& Viikari, J. (1997). Physical activity in childhood and adolescence as predictor of physical activity in young adulthood. American Journal of Preventive Medicine, 13, 317-23.

Tsourlou, T., Benik, A., Dipla, K., Zafeiridis, A., \& Kellis, S. (2006). The effects of a twenty-four-week aquatic training program on muscular strength performance in healthy elderly women. Journal of Strength and Conditioning Research, 20(4), 811. https://doi.org/10.1519/R-18455.1. 